\title{
Threshold Selection for POT Framework in the Extreme Vehicle Loads Analysis Based on Multiple Criteria
}

\author{
Guangrun Wu $(\mathbb{D})$ and Wenliang Qiu \\ School of Civil Engineering, Dalian University of Technology, Dalian 116024, China \\ Correspondence should be addressed to Wenliang Qiu; qwl@dlut.edu.cn
}

Received 10 November 2017; Revised 22 January 2018; Accepted 5 March 2018; Published 11 April 2018

Academic Editor: Roberto Palma

Copyright (C) 2018 Guangrun Wu and Wenliang Qiu. This is an open access article distributed under the Creative Commons Attribution License, which permits unrestricted use, distribution, and reproduction in any medium, provided the original work is properly cited.

\begin{abstract}
Extreme value of vehicle load plays an important role in bridge design and risk assessment. Peaks over threshold (POT) is a method commonly used in extreme load estimation. The selection of thresholds for the POT method is extremely crucial, but the selected optimal threshold varies under different test criteria. Therefore, a method to select the suitable threshold is developed based on multiple criteria decision analysis (MCDA) in the paper. In MCDA, Chi-Square $\left(\chi^{2}\right)$ test, Kolmogorov-Smirnov $(\mathrm{K}-S)$ test, and Root Mean Square Error (RMSE) in probability distribution functions (PDF) are employed as the test criteria and the weight of these criteria is calculated using the entropy method. Finally, vehicle loads obtained from simulation and field measurement are adopted to validate the effectiveness and feasibility of the proposed method. The results indicate that the proposed MCDA is a useful and complementary tool for threshold selection in the extreme value analysis.
\end{abstract}

\section{Introduction}

Vehicle load is one of the most significant factors for bridge design, safety assessment, and fatigue analysis [1-5]. Overloaded heavy vehicles is the primary reason for the deterioration of structural components and the degradation of the bridge's overall state [6-8]. The condition of road traffic has gained a notable increase during the service period due to the rapid economic growth. Many bridge collapse accidents have resulted from overloading vehicles. In addition, the number of bridges worldwide stepping into their ageing stage is growing, which raises the risk of structural safety for users. It is extremely expensive to rehabilitate or maintain the degraded bridge due to the project itself, traffic disruption, and resulting delays. Large amounts of costs associated with rehabilitation and maintenance can be saved by proving that the bridge is safe through accurate risk assessment or proposing an appropriate repair scheme [9]. Therefore, it is necessary to have a good estimation of vehicle loads, and this typically involves using Extreme Value Theory (EVT).

Collecting the actual load time history is time consuming and costly. Generally, the extreme loads among the design life of the bridge cannot be measured through limited period of data. The EVT can solve the problem through the estimation of the extreme vehicle loads from a relative short period to a long period. The EVT is used to quantify the stochastic behavior of a process at unusually large or small levels. Most of the published works about the EVT applied to vehicle loads are on the basis of the block maximum (BM) and POT methods [10]. The BM method divides the observation period into nonoverlapping periods of equal size. Then the largest value in each period is extracted to generate maximum sample, which can be fitted by generalized extreme value (GEV) distribution. Using the BM method to extreme value estimation requires a large quantity of data, and thus it is not appropriate to use this method when the available data is insufficient. An alternative to the BM method is peak over threshold (POT) method. The POT method extracts the peak values exceeding a threshold, and the extracted data can be fitted by generalized Pareto distribution (GPD).

A well-known problem for the POT method is to select a suitable threshold [11]. Threshold selection is the trickiest part of the POT method because only a narrow range of thresholds is applicable. On the one hand, a considerable high threshold is required to guarantee that GPD parameters are stable. On the other hand, the threshold level cannot be too 
high so that sufficient data can be included [12]. The literature suggests that the most used threshold selection methods are based on judgement [13]. One way is to use graphical diagnostic plots, but interpreting these plots is subjective and rather challenging. The other way is to select a fixed quantile threshold corresponding to a high nonexceedance probability. The drawback of this method is that once the threshold has been determined, it is treated as fixed and the associated subjectivity and uncertainty are ignored in subsequent inferences.

In contrast to the above methods, the numerical approaches are relatively objective for threshold election, which are conducted based on goodness-of-fit tests across a range of different thresholds. There are many popular criteria for the goodness-of-fit tests to measure the distance between the cumulative distribution function of the reference distribution and the empirical distribution of the sample, such as Root Mean Square Error (RMSE) test, Kolmogorov-Smirnov (K-S) test, Pearson's Chi-Square $\left(\chi^{2}\right)$ test, and Anderson-Darling (A-D) test. It is observed that the selected threshold under one test criterion may not be the optimal threshold for other criteria. The performance of extreme value estimation is greatly affected when the extracted sample changes due to the test criterion variation. To select an optimal and stable threshold for the POT method, it is meaningful to do some research to take multiple criteria into consideration. The multiple criteria decision analysis (MCDA) approach works when practical problems are characterized by several conflicting criteria [14]. An entropy method is employed to assign the weight value of each criterion for the MCDA, and then the MCDA can integrate results obtained from goodness-of-fit under different criteria into a comprehensive one, which makes the selection more scientific and objective [15].

In this paper, the MCDA is proposed to select a threshold for the POT method in the extreme value analysis of vehicle loads. Section 2 presents the theory of the POT method, the mathematical methodologies of MLE, and the framework of extreme loads estimation. Section 3 presents details of the MCDA approach involving test criteria and the maximum entropy theory. Sections 4 and 5 present a practical application of theories illustrated in Sections 2 and 3 on simulated and field monitored vehicle loads. Finally, Section 6 presents some conclusions of this paper.

\section{Theory of Extreme Value Analysis}

2.1. The POT Method. Figure 1 shows the POT method of how to extract extreme values from the observations, where $\mu$ is the threshold. The distribution of the entire observations can be treated as a combination of the body part and the tail part. Under the POT framework, our focus is the observations exceeding the threshold, not the entire observations. When the values of the observations exceed the threshold, these observations are called exceedances $X\left(x_{1}, x_{2}, \ldots, x_{n}\right)$. Supposing $X$ is a sequence of independent and identically distributed random variables belonging to a continuous distribution function, that is, $F(x)$. The exceedances above the

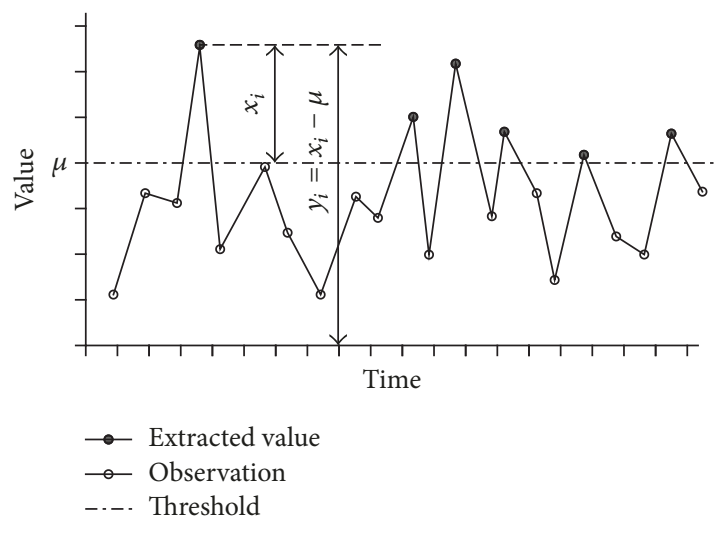

FIGURE 1: Illustration of the POT method.

threshold $\mu$ are modelled by $y_{i}=x_{i}-\mu$, and the distribution function of $F_{\mu}(y)$ is expressed as follows:

$$
\begin{array}{r}
F_{\mu}(y)=P\{X-\mu \leq y \mid X>\mu\}=\frac{F(y+\mu)-F(\mu)}{1-F(\mu)}, \\
y>0 .
\end{array}
$$

When $\mu$ is sufficiently large, the distribution function of $F_{\mu}(y)$ approximately obeys the GPD, whose cumulative distribution function is defined in

$$
F(y)= \begin{cases}1-\left[1+\frac{\xi y}{\sigma}\right]^{-1 / \xi}, & \xi \neq 0, \\ 1-\exp \left(-\frac{y}{\sigma}\right), & \xi=0,\end{cases}
$$

where $\xi$ is the shape parameter of the distribution, $\sigma$ is the scale parameter of the distribution, and $\theta$ is the vector $(\sigma, \xi, \mu)$. Often, the threshold $\mu$ is supposed to be known prior to parameters estimation and the GPD is specified only by parameters $\sigma$ and $\xi$. The GPD is divided into three types depending on the value of shape parameter $\xi: F(y)$ is shorttailed when $\xi<0 ; F(y)$ is medium-tailed when $\xi=0$; and $F(y)$ is heavy-tailed when $\xi>0$. The estimation of extreme vehicle loads is calculated through return level analysis. In the GPD model, the return value of the $T$-year return period $y_{T}$ can be obtained as follows:

$$
\begin{aligned}
y_{T} & =\mu+\frac{\sigma}{\xi}\left[(\lambda T)^{\xi}-1\right], \quad \xi \neq 0, \\
y_{T} & =\mu+\sigma \log (\lambda T), \quad \xi=0, \\
\lambda & =\frac{n_{u}}{n},
\end{aligned}
$$

where $n$ is the number of observations and $n_{u}$ is the number of observations exceeding the threshold.

2.2. MLE Method. Calculating the parameters of the GPD is another decisive issue in the extreme value analysis. The maximum likelihood estimation (MLE) method can balance the observed data and possible prediction [16]. When the 
distribution is known, the MLE method is commonly used to calculate the unknown parameters of the distribution with advantages of asymptotic normality, invariance, and congruency. The MLE method is based on the likelihood function as follows:

$$
L(y ; \theta)=\prod_{i=1}^{n} f\left(y_{i} ; \theta\right),
$$

where $f\left(y_{i} ; \theta\right)$ is the PDF of $i$ th observed variable $y_{i}$ and $n$ is the number of the extracted samples. In practice, the logarithmic form is more convenient to perform. The logarithmic form of the likelihood function takes

$$
\ln L(y ; \theta)=\prod_{i=1}^{n} \ln f\left(y_{i} ; \theta\right)
$$

When the extracted sample is obtained, the likelihood function $\ln L(y ; \theta)$ becomes the function of the variable $\theta$. The maximum value of $\ln L(\theta)$ occurs when $\mathrm{d} \ln L(\theta) / \mathrm{d}(\theta)=0$ and the variable $\theta$ is determined.

$$
\frac{\mathrm{d} \ln L\left(y_{i} ; \theta\right)}{\mathrm{d} \theta}=0 .
$$

Substituting (5) into (6), (7) can be obtained. The parameters of the GPD are obtained by solving

$$
\begin{gathered}
\sum_{i=1}^{n} \ln \left(1+\xi \frac{y_{i}}{\sigma}\right)=\xi n, \\
(1+\xi) \sum_{i=1}^{n} \xi \frac{y_{i}}{\sigma+\xi y_{i}}=n .
\end{gathered}
$$

2.3. Flowchart of Extreme Loads Estimation. Figure 2 illustrates the flowchart of the extreme vehicle loads estimation. As illustrated in Figure 2, once a value is set as candidate threshold, the corresponding GPD fitting can be carried out. The accuracy of the GPD fitting is checked by goodness-of-fit tests under multiple criteria, and then the optimal threshold is determined based on MCDA.

The key point of the flowchart is MCDA, whose process contains four main stages including scope determination of candidate thresholds, criteria selection, criteria weight determination, and threshold examination. According to the character of the load distribution, candidate thresholds $\left(\mu_{1}, \ldots\right.$, $\mu_{m}$ ) are set in ascending order, where $\mu_{1}$ is the relatively low quantile of the load data value and $\mu_{m}$ is the $99 \%$ quantile of the load data value. Three popular test criteria are adopted in the MCDA, and the weight of each criterion is determined based on the maximum entropy theory. The maximum entropy method is an objective weight-determining method because the criterion that has the greatest difference will take the largest weight in decision-making $[15,17]$. To validate the reliability of the proposed method of the threshold selection, the graphical quantile-quantile (Q-Q) plot is performed. Finally, the estimation of extreme vehicle loads is conducted through return level analysis.

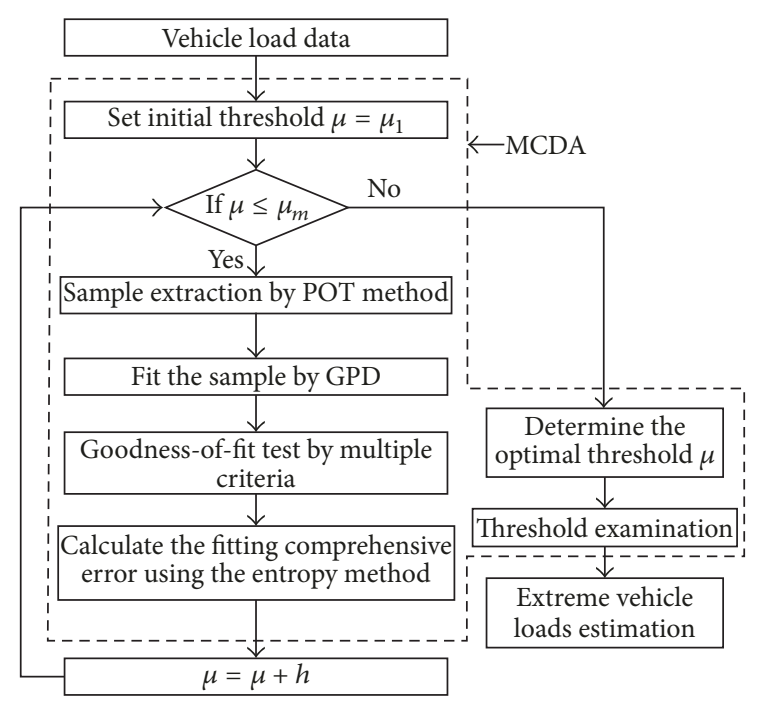

FIGURE 2: Flowchart of the extreme vehicle loads estimation.

\section{Methodology of the Threshold Selection}

3.1. Test of Goodness-of-Fit. There are nearly 40 test criteria in the statistical literature, and the principles of these criteria are different. The most popular methods are Chi-Square test $\left(\chi^{2}\right)$, Kolmogorov-Smirnov (K-S) test, Anderson-Darling (A-D) test, Root Mean Square Error (RMSE) test, and correlation coefficient test. To examine the fitting effect of the GPD in the MCDA, three commonly used test criteria, the K-S test, $\chi^{2}$ test, and RMSE test, are adopted. The details of these tests are shown as follows:

$$
\begin{aligned}
K_{\max } & =\max _{1 \leq j \leq n-1}\left\{\left|F\left(x_{i}\right)-G\left(x_{i}\right)\right|,\left|F\left(x_{i+1}\right)-G\left(x_{i}\right)\right|\right\}, \\
\chi^{2} & =\sum_{i=1}^{n} \frac{\left(O_{i}-Q_{i}\right)^{2}}{E_{i}} \\
\delta_{\mathrm{pdf}} & =\frac{1}{n^{2}} \sum_{i=1}^{n}\left(f\left(x_{i}\right)-g\left(x_{i}\right)\right)^{2} .
\end{aligned}
$$

The test value $K_{\max }$ measures the greatest deviation between the theoretical CDF and the empirical CDF; the test value $\chi^{2}$ computes the deviation between the observed frequency $O_{i}$ and the expected frequency $Q_{i}$; and the test value $\delta_{\text {pdf }}$ calculates the root mean deviation between the theoretical PDF and the empirical PDF. Among the three tests, the smaller the test values $K_{\max }, \chi^{2}$, and $\delta_{\text {pdf }}$, the better the effect of the fitting distribution obtained.

3.2. Entropy Method. For each candidate threshold, a test value will be calculated under the corresponding test criterion. For a series of candidates under multiple test criteria, there will be many test values. Each of them has its role in the evaluation of fitting accuracy. To find a suitable threshold under multiple criteria, these criteria should be considered simultaneously. Considering the different principles of the test criteria, the greater the weight value is, the more important the test criterion will be. The weight values of the criteria 
should be settled objectively, and therefore the maximum entropy method is used.

Given that the tests are achieved by a range of candidate thresholds, each threshold belongs to a criterion. The number of candidate thresholds is $m$, the number of test criteria is $n$, and $r_{i j}$ represents the performance value of the $i$ th candidate threshold to the $j$ th criterion from the decision matrix $R$

$$
R=\begin{array}{cccc}
w_{1} & w_{2} & \cdots & w_{n} \\
C_{1} & C_{2} & \cdots & C_{n} \\
L_{1} \\
L_{2} \\
\vdots \\
L_{m}
\end{array}\left[\begin{array}{cccc}
r_{11} & r_{12} & \cdots & r_{1 n} \\
r_{21} & r_{12} & \cdots & r_{2 n} \\
\vdots & \vdots & \ddots & \vdots \\
r_{m 1} & r_{m 2} & \cdots & r_{m n}
\end{array}\right],
$$

where the vectors $\left(C_{1}, C_{2}, \ldots, C_{n}\right)$ represent the criteria and the alternatives $\left(L_{1}^{T}, L_{2}^{T}, \ldots, L_{m}^{T}\right)$ represent the candidate thresholds. The vector $\left(w_{1}, w_{2}, \ldots, w_{n}\right)$ represents the importance and relative weights of the chosen criteria, which can be obtained from the decision matrix. To show the weight values of different criteria more directly, the decision matrix $R$ is transformed to

$$
p_{i j}=\frac{r_{i j}}{\sum_{i=1}^{m} r_{i j}} .
$$

Then the normalized decision matrix $P$ is obtained from (10) as follows:

$$
P=\left[\begin{array}{llll}
p_{11} & p_{12} & \cdots & p_{1 n} \\
p_{21} & p_{22} & \cdots & p_{2 n} \\
p_{31} & p_{32} & \ddots & p_{3 n} \\
p_{m 1} & p_{m 2} & \cdots & p_{m n}
\end{array}\right]
$$

As a useful tool to describe the relative weights of the criteria, the $j$ th entropy value is defined by $E_{j}$ and obtained from the following formulation:

$$
E_{j}=-\frac{1}{\ln (m)} \sum_{i=1}^{m} p_{i j} \ln \left(p_{i j}\right) \quad j=1,2, \ldots, n .
$$

Note that there is an inverse relationship between $E_{j}$ and $w_{j}$. To ensure (13), $E_{j}$ is substituted with $1-E_{j}$ and normalized for direct illustration

$$
\sum_{j=1}^{n} w_{j}=1, \quad 0 \leq w_{j} \leq 1,
$$

where $w_{j}$ is the objective weight value of the $j$ th criterion. The most reasonable expression of normalized weight value is defined by

$$
w_{j}=\frac{\left(1-E_{j}\right)}{\sum_{j=1}^{n}\left(1-E_{j}\right)} .
$$

The normalized weight value of different criteria can be obtained from
TABLE 1: Statistical parameters of vehicle loads.

\begin{tabular}{lccc}
\hline Type & $u_{\ln G}(\mathrm{~kg})$ & $\sigma_{\ln G}(\mathrm{~kg})$ & $p(\%)$ \\
\hline I & 7.65 & 0.36 & 63.48 \\
II & 8.84 & 0.67 & 4.84 \\
III & 9.51 & 0.39 & 24.89 \\
IV & & & 6.79 \\
\hline
\end{tabular}

$$
W=\left[w_{1}, w_{2}, \ldots, w_{n}\right]^{T}
$$

Finally, the comprehensive evaluation indicator can be calculated considering multiple test criteria by (16). When the GPD fitting is performed, the smaller the comprehensive evaluation indicator, the better the fitting effect of goodnessof-fit obtained

$$
S=R W \text {. }
$$

\section{Simulation Study}

To validate the application and feasibility of the proposed MCDA method, a simulation study is carried out. The advantage of the simulation is to produce a required mixture distribution where the tail part obeys the GDP distribution and the threshold is known. Referring to the probabilistic vehicle load models (Yang et al. 2014), the proportions and statistical parameters of different kinds of vehicles are shown in Table 1 . The body part of the mixture distribution in the simulation includes two-axle vehicle loads of light vehicles (Type I), heavy vehicles (Type II), and trucks (Type III). The two-axle vehicle loads obey the log-normal distributions as expressed in (17), where $g$ is the random variable of the vehicle's gross weight and $\mu_{\ln (G)}$ and $\sigma_{\ln (G)}$ are the mean value and the standard deviation of logarithmic values of vehicle loads. The tail part of the mixture distribution in the simulation includes multiple-axle vehicles (Type IV). The multiple-axle vehicle loads obey the GPD, and the parameters are set as $\xi=0.001, \sigma=6$, and $\mu=17.5$. According Table 1 , $10 e 5$ simulated vehicle loads are generated, and their mixture distribution is shown in Figure 3. The two-axle vehicle loads in the body part range from 0 to $17.5 \mathrm{t}$ and the multiple-axle vehicle loads in the tail part are above the threshold $17.5 \mathrm{t}$, and the threshold of the mixture distribution is $17.5 \mathrm{t}$

$$
f_{G}(g)=\frac{1}{\sqrt{2 \pi} \sigma_{\ln (G)} g} \exp \left[-\frac{1}{2}\left(\frac{\ln (g)-\mu_{\ln (G)}}{\sigma_{\ln (G)}}\right)\right] .
$$

A total of 201 candidate thresholds are set from the interval [10 30], and the GPD of each threshold is fitted to the extracted samples of extreme values, respectively. The goodness-of-fit tests are performed based on single criterion and multiple criteria, and the results are shown in Figure 4. It can be seen from Figure 4(a) that the statistics can reach extremum rather than minimum when the candidate threshold is 17.5 t. $K_{\max }$ reaches its minimum when the threshold is $20.3 \mathrm{t}, \chi^{2}$ reaches its minimum when the threshold is $21.6 \mathrm{t}$, and $\delta_{\text {pdf }}$ reaches its minimum when the threshold is $16.1 \mathrm{t}$. Based on the maximum entropy method, the objective weight 


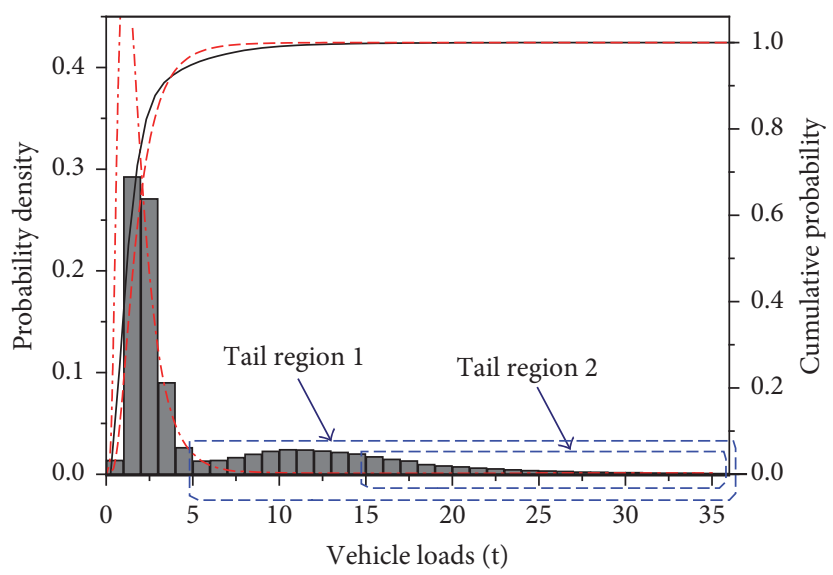

Empirical PDF _- $\quad$ Theoretical PDF
Empirical CDF

(a)

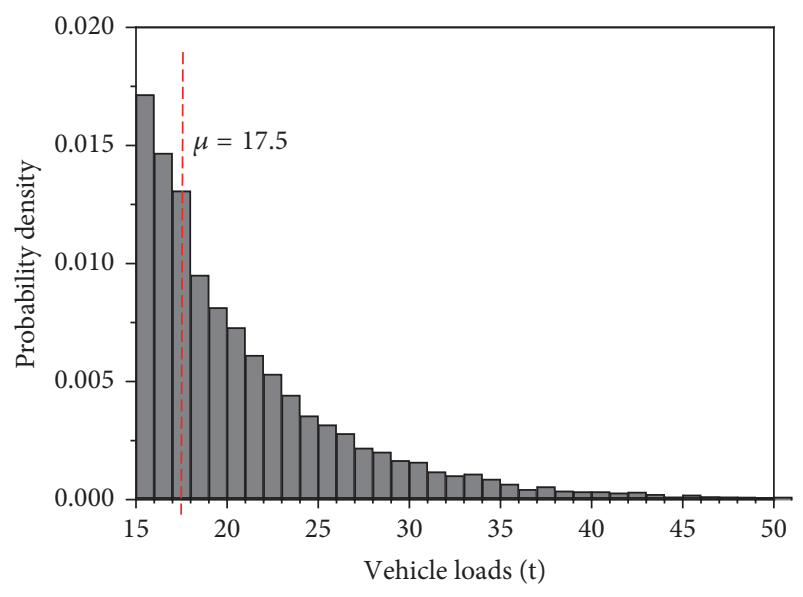

(b)

FIGURE 3: Mixture distribution of the simulated data: (a) entire range and (b) tail region.

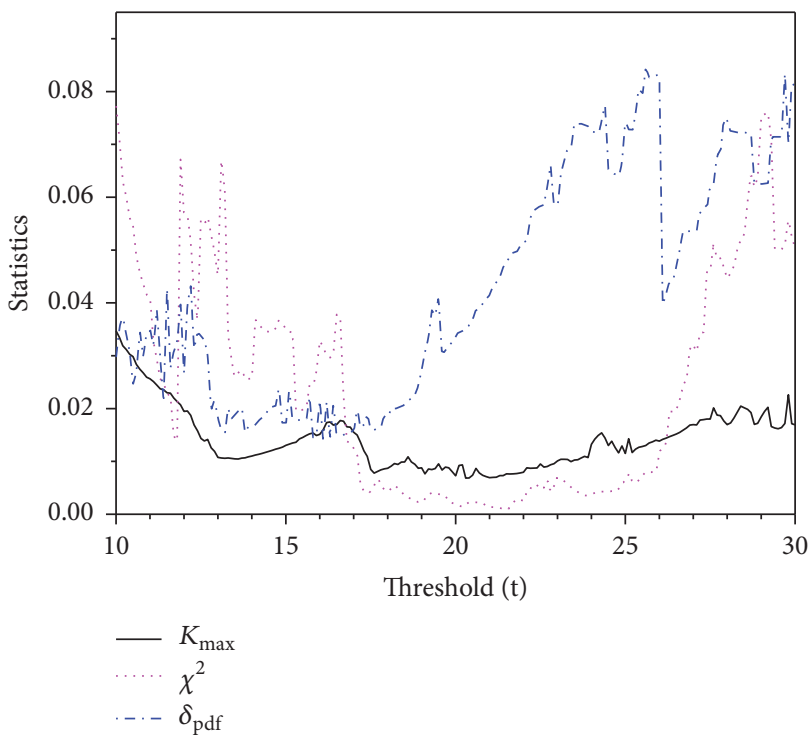

(a)

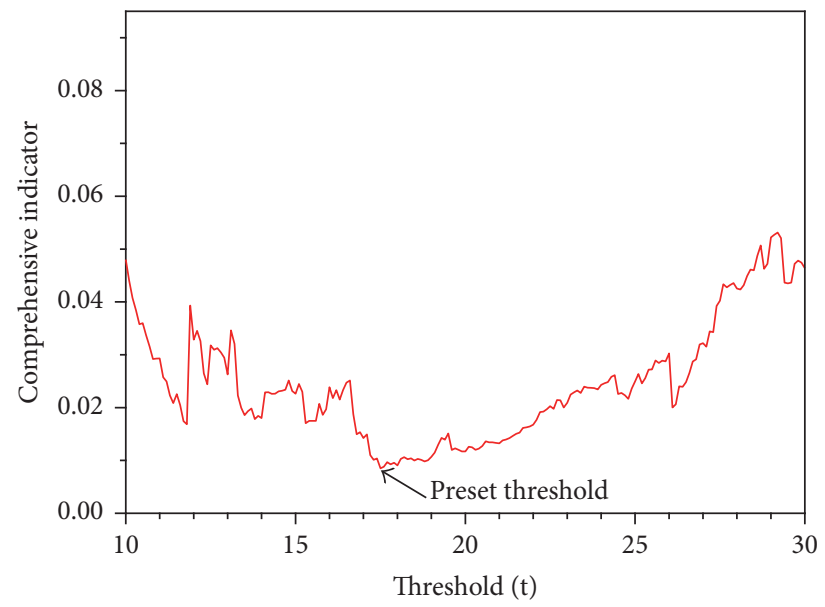

(b)

Figure 4: Goodness-of-fit test: (a) single criteria and (b) multiple criteria.

values of the statistics $K_{\max }, \chi^{2}$, and $\delta_{\text {pdf }}$ are calculated: $w=$ $[0.2613,0.3812,0.3575]$. Then the comprehensive indicator is calculated. It can be concluded from Figure 4(b) that the comprehensive evaluation indicator reaches the minimum value when the threshold is $17.5 \mathrm{t}$ and that the value is the same with the preset threshold of the mixture distribution.

\section{Case Study}

5.1. Fit the Distribution Using Traditional Approach. Figure 5 shows the real image of the Dalian Northern Suspension Bridge. The bridge, opened to public in May 1987, is the

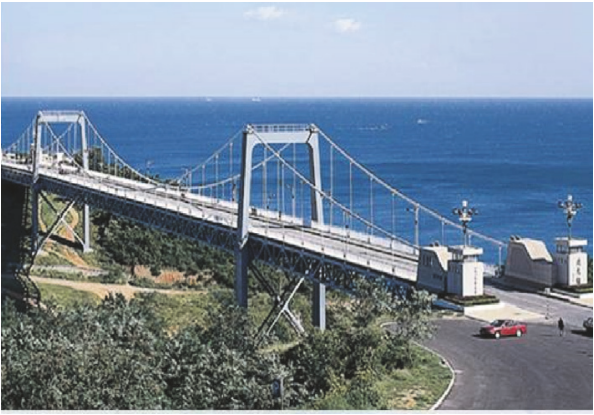

Figure 5: Dalian Northern Suspension Bridge. 


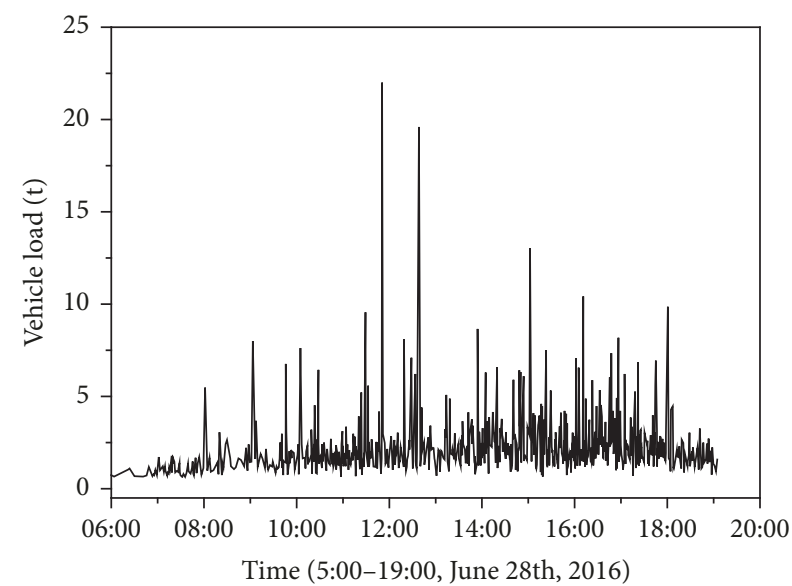

(a)

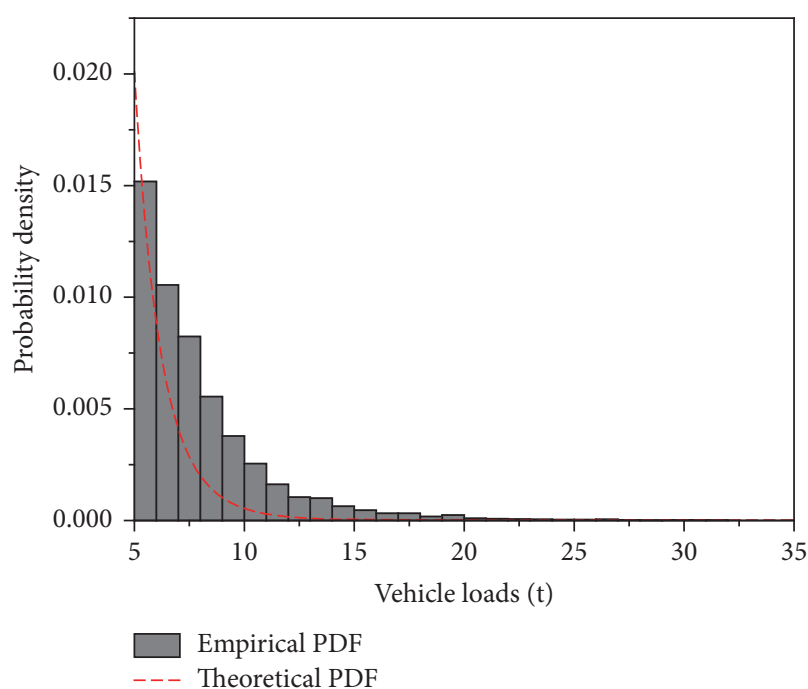

(c)

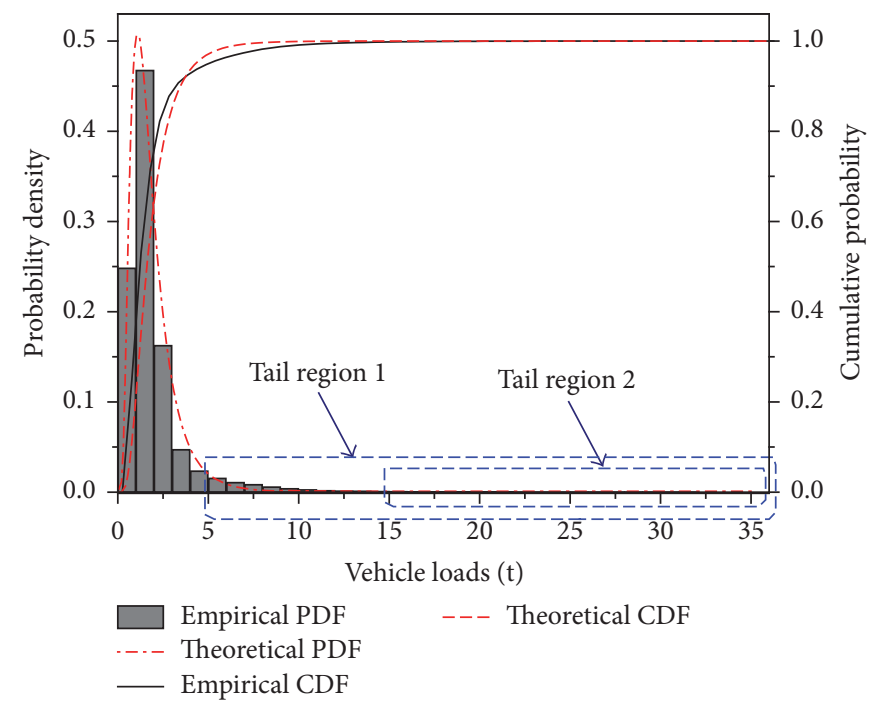

(b)

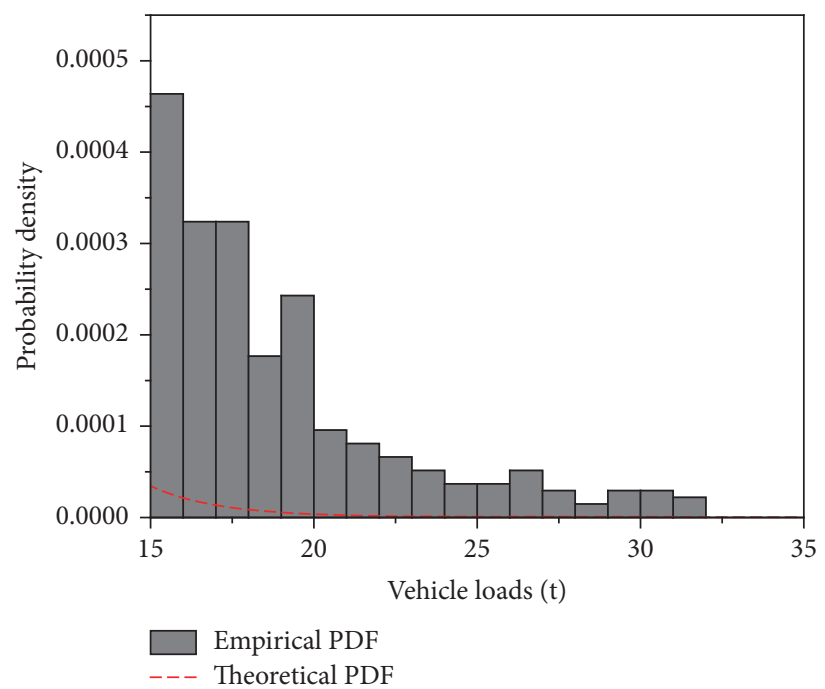

(d)

FIGURE 6: Time history and log-normal fit of the vehicle load: (a) actual load history of vehicle load, (b) entire ranger, (c) tail region 1, and (d) tail region 2.

first three-span suspension bridge in China with simply supported stiffening trusses and earth anchorage system. The bridge has two pedestrian lanes and two vehicular lanes that can carry heavy traffic, which played a significant role in the coastal highway of Dalian. Restricted to the road width, multiple-axle wheeled vehicles are not allowed to pass through the bridge. During the rehabilitation in April 2016, a sophisticated online SHM system was designed and equipped in the suspension bridge. The SHM system incorporates a WIM system, which is located at the north side of the bridge to measure the traffic load. The measured vehicle loads of the suspension bridge from June 2016 to June 2017 are employed to illustrate the proposed method of threshold selection for the POT framework in the extreme vehicle load analysis.

Figure 6 shows the time history of the actual vehicle load and its statistical distribution. The vehicle load of the Dalian
Northern Suspension Bridge has been continuously observed since the installation of the SHM system, and a representative example of the measured load history is shown in Figure 6(a). The entire range and tail region of the statistical distribution of vehicle loads are illustrated in Figures 6(b)-6(d). The theoretical fitting to the empirical data uses log-normal distribution, which is recommend by Unified Standard for Reliability Design of Highway Engineering Structures (GB/T 50283-1999). It can be seen from Figures 6(c) and 6(d) that the fitting effect is not ideal as the theoretical distribution deviates considerably from the empirical distribution, especially in the tail region.

5.2. Threshold Selection Using MCDA. The measured vehicle loads range from $0.14 \mathrm{t}$ to $32 \mathrm{t}$, a total of 281 candidate thresholds from $2 \mathrm{t}$ to $30 \mathrm{t}$ are set as the intervals, and the 


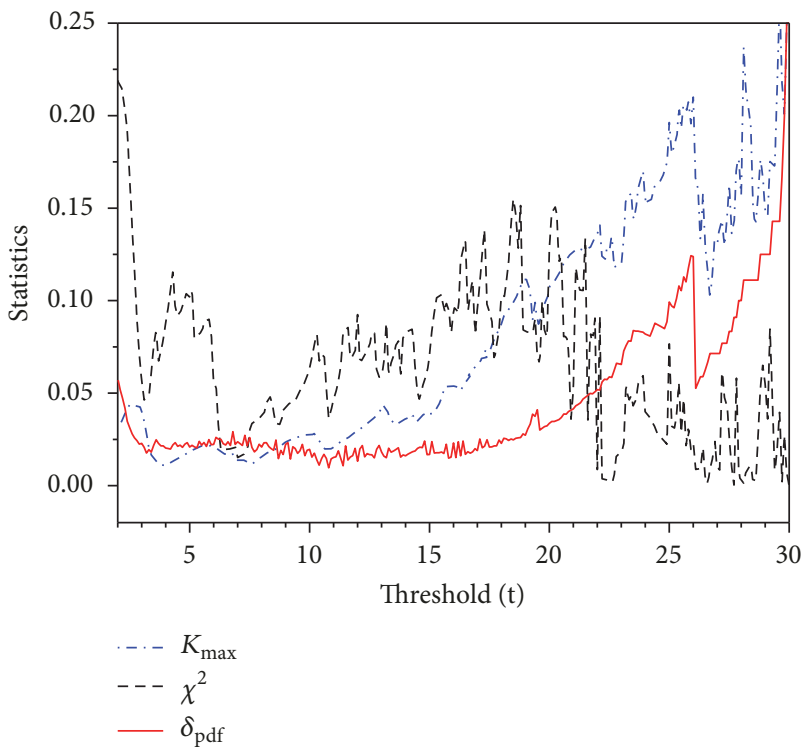

(a)

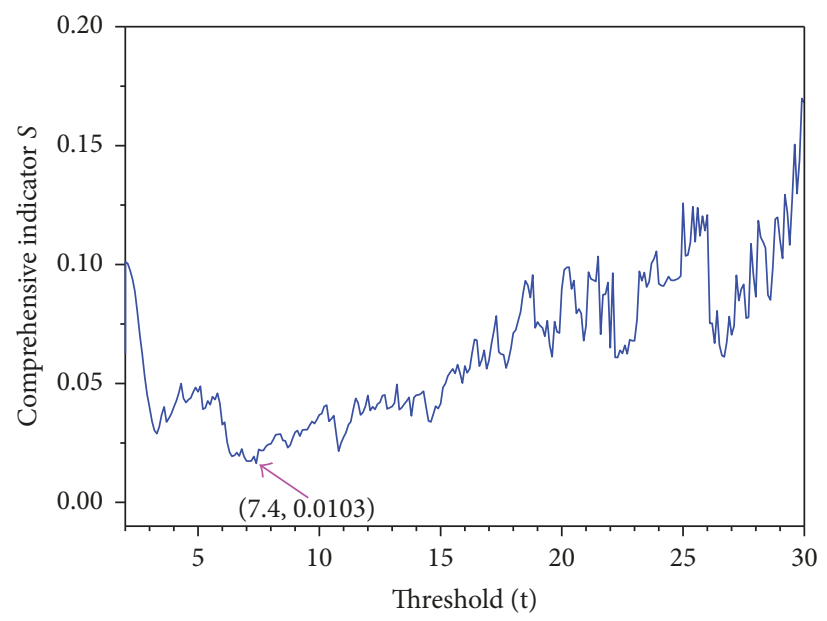

(b)

FIGURE 7: Goodness-of-fit test: (a) single criterion and (b) multiple criteria.

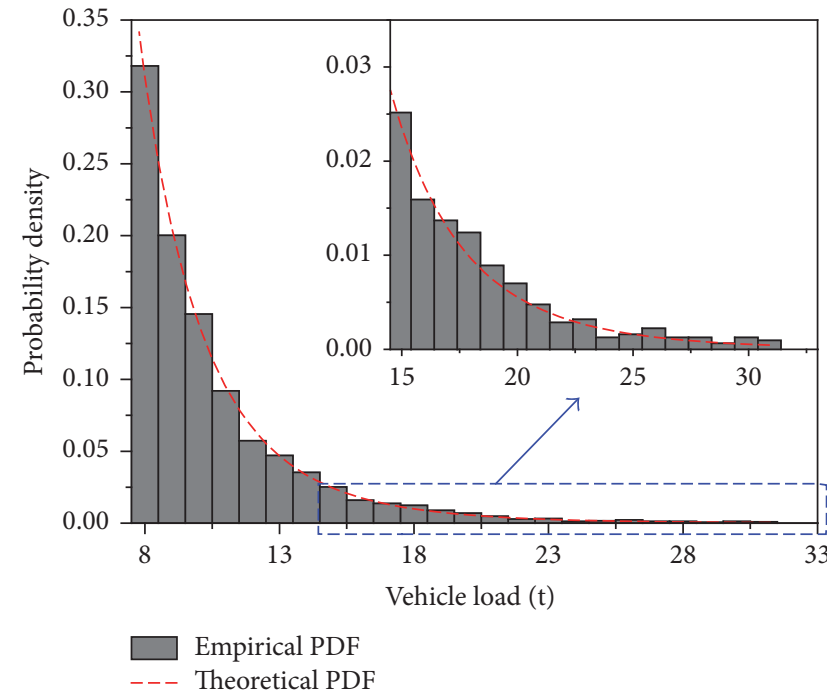

(a)

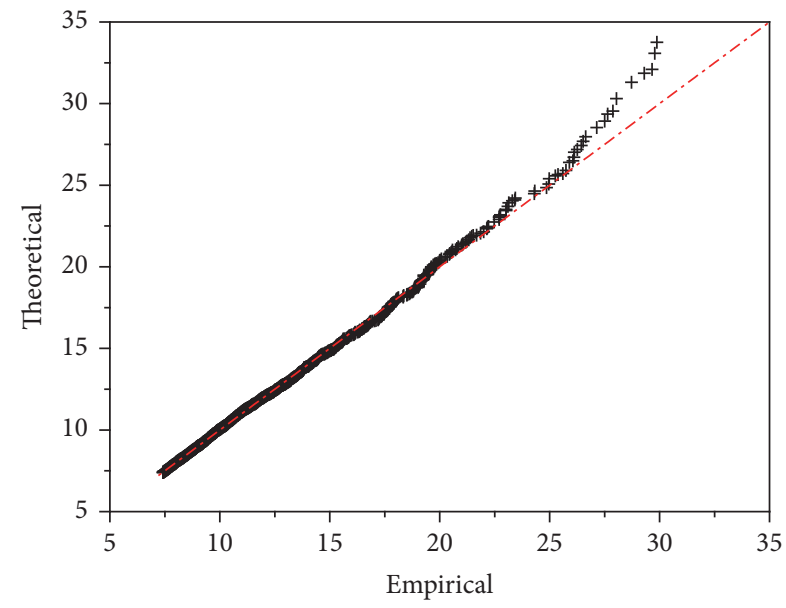

(b)

FIGURE 8: GPD fitting: (a) entire range above the threshold and (b) graphical Q-Q plot.

GPD fitting under each candidate threshold is conducted after the POT process, respectively. Figure 7 shows the statistics of $K_{\max }, \chi^{2}, \delta_{\mathrm{pdf}}$, and their comprehensive evaluation indicator. It can be seen from Figure 7(a) that the selected thresholds are not consistent under different test criteria and the curve trends for different test criteria are quite different. $K_{\max }$ reaches its minimum when the threshold is $3.6 \mathrm{t} ; \chi^{2}$ reaches its minimum when the threshold is $22.6 \mathrm{t}$; and $\delta_{\text {pdf }}$ reaches its minimum when the threshold is $10.9 \mathrm{t}$. Based on the maximum entropy method, the objective weight values of the statistics $K_{\max }, \chi^{2}$, and $\delta_{\text {pdf }}$ are calculated: $w=[0.3417,0.3281,0.3302]$, and then the comprehensive evaluation indicator is obtained as shown in Figure 7(b). It can be concluded from Figure 7(b) that the comprehensive evaluation indicator reaches the minimum value when the threshold is $7.4 \mathrm{t}$. The statistics of $K_{\max }, \chi^{2}$, and $\delta_{\mathrm{pdf}}$ reach their extremum when the threshold is $7.4 \mathrm{t}$, and this value is selected as the threshold for extreme load estimation.

5.3. GPD Fitting and Extreme Load Estimation. After selecting a reasonable threshold, the monitored vehicle loads are processed through the POT framework and the extracted extreme values are fitted to the GPD. Figure 8 shows the GPD fitting of extracted extreme vehicle loads above the selected threshold and its graphical Q-Q plot. It can be seen from Figure 8 (a) that the fitting accuracy of extreme vehicle loads 


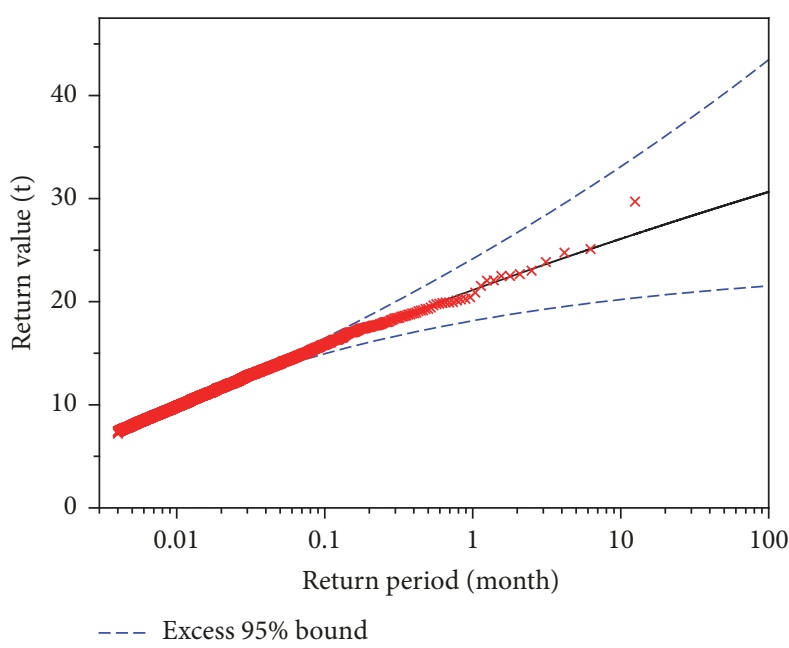

(a)

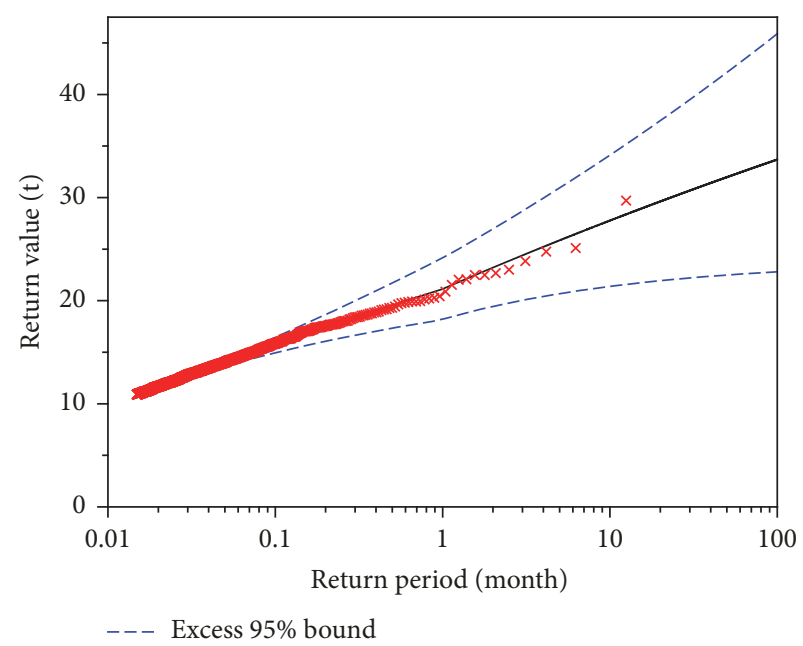

(b)

Figure 9: Return level analysis: (a) $\mu=7.4 \mathrm{t}$ and (b) $\mu=10.9 \mathrm{t}$.

has been improved considerably compared with traditional approach. When the fitting effect is satisfactory, most empirical observation values excess threshold are scattered near the line in the Q-Q plot. It can be seen from Figure 8(b) that most empirical observations can scatter near the fitting line and there is only a small difference between fitted values and empirical values in the $\mathrm{Q}-\mathrm{Q}$ plot.

To estimate the extreme vehicle loads and validate the fitting effect of GPD, return level analysis is carried out. Figure 9 shows the return level analysis of the vehicle loads under the thresholds $\mu=7.4 \mathrm{t}$ and $\mu=10.9 \mathrm{t}$. It can be from Figure 9 that the empirical observations over the selected threshold are located within 95\% confidence interval of the estimated levels. When the return period is 100 months, the return values are $30.7 \mathrm{t}$ and $33.7 \mathrm{t}$ under the threshold $\mu=7.4 \mathrm{t}$ and $\mu=10.9 \mathrm{t}$, which indicates that the estimated values of extreme load are in a more reasonable range because heavy multiple-axle wheeled vehicles are restricted. The results convince us that the GPD fitting effect is relatively satisfactory and the selected threshold is reasonable.

\section{Conclusions}

When estimating extreme values of vehicle loads through the POT method, threshold selection is the key point because the chosen threshold is sensitive to the extracted extreme loads sample and parameter estimation. To model a reliable extreme distribution by reducing the fitting error of the GPD and evaluate the selected threshold, a comprehensive evaluation approach based on MCDA is proposed in this paper. It is a new trial considering multiple criteria in threshold selection, and the weight values are calculated by using the maximum entropy theory. The optimal threshold is determined through a range of candidate thresholds automatically and fitting results are analyzed quantitatively.

The simulated data and field measurement of vehicle loads were adopted to validate the practicability and superiority of the proposed method, respectively. The results illustrate that the MCDA method proposed in this paper reduces subjectivity during threshold selection, which can be flexibly used in various situations. The proposed MCDA can help select a suitable threshold for the POT framework, and it exhibits a better estimation of extreme vehicle loads through return level analysis with the selected threshold. In practice, the proposed MCDA is a useful tool in the extreme value analysis that complements the existing methods of thresholds selection.

\section{Conflicts of Interest}

There are no conflicts of interest related to this paper.

\section{Acknowledgments}

This presented research was financially supported by the National Natural Science Foundation of China (Grant no. 51778108).

\section{References}

[1] A. Getachew and E. J. Obrien, "Simplified site-specific traffic load models for bridge assessment," Structure and Infrastructure Engineering, vol. 3, no. 4, pp. 303-311, 2007.

[2] D. M. Frangopol, A. Strauss, and S. Kim, "Bridge reliability assessment based on monitoring," Journal of Bridge Engineering, vol. 13, no. 3, pp. 258-270, 2008.

[3] S. G. M. Neves, A. F. M. Azevedo, and R. Calçada, "A direct method for analyzing the vertical vehicle-structure interaction," Engineering Structures, vol. 34, pp. 414-420, 2012.

[4] D. Sun, X. Wang, B. Chen, and B. Sun, "Probability model of hangzhou bay bridge vehicle loads using weigh-in-motion data," Shock and Vibration, vol. 2015, Article ID 576083, 10 pages, 2015.

[5] M. Soriano, J. R. Casas, and M. Ghosn, "Simplified probabilistic model for maximum traffic load from weigh-in-motion data," Structure and Infrastructure Engineering, vol. 13, pp. 454-467, 2017. 
[6] G. Fu, L. Liu, and M. D. Bowman, "Multiple presence factor for truck load on highway bridges," Journal of Bridge Engineering, vol. 18, no. 3, pp. 240-249, 2011.

[7] W. Qiu, M. Jiang, and C. Huang, "Parametric study on responses of a self-anchored suspension bridge to sudden breakage of a hanger," The Scientific World Journal, vol. 2014, Article ID 512120, 10 pages, 2014.

[8] X. Shi, Z. Zhou, and X. Ruan, "Failure analysis of a girder bridge collapse under eccentric heavy vehicles," Journal of Bridge Engineering, vol. 21, Article ID 05016009, 2016.

[9] M. Liu and D. M. Frangopol, "Optimizing bridge network maintenance management under uncertainty with conflicting criteria: Life-cycle maintenance, failure, and user costs," Journal of Structural Engineering, vol. 132, no. 11, Article ID 016611QST, pp. 1835-1845, 2006.

[10] P. Thompson, Y. Cai, D. Reeve, and J. Stander, "Automated threshold selection methods for extreme wave analysis," Coastal Engineering Journal, vol. 56, no. 10, pp. 1013-1021, 2009.

[11] A. Tancredi, C. Anderson, and A. O'Hagan, "Accounting for threshold uncertainty in extreme value estimation," Extremes, vol. 9, no. 2, pp. 87-106, 2006.

[12] C. Scarrott and A. MacDonald, "A review of extreme value threshold estimation and uncertainty quantification," $R E V$ STAT-Statistical Journal, vol. 10, no. 1, pp. 33-60, 2012.

[13] C. Caballero-Megido, J. Hillier, D. Wyncoll, L. Bosher, and B. Gouldby, "Technical note: Comparison of methods for threshold selection for extreme sea levels," Journal of Flood Risk Management, 2017.

[14] H. Çalışkan, B. Kurşuncu, C. Kurbanoğlu, and Ş. Y. Güven, "Material selection for the tool holder working under hard milling conditions using different multi criteria decision making methods," Materials \& Design, vol. 45, pp. 473-479, 2013.

[15] J.-J. Wang, Y.-Y. Jing, C.-F. Zhang, and J.-H. Zhao, "Review on multi-criteria decision analysis aid in sustainable energy decision-making," Renewable \& Sustainable Energy Reviews, vol. 13, no. 9, pp. 2263-2278, 2009.

[16] J. R. Hosking and J. R. Wallis, "Parameter and quantile estimation for the generalized pareto distribution," Technometrics, vol. 29, no. 3, pp. 339-349, 1987.

[17] J. Wang, N. Wang, Z. Wang, Y. Zhang, and L. Liu, "Determination of the minimum sample size for the transmission load of a wheel loader based on multi-criteria decision-making technology," Journal of Terramechanics, vol. 49, no. 3-4, pp. 147160, 2012. 


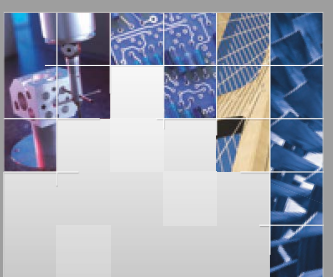

\section{Enfincering}
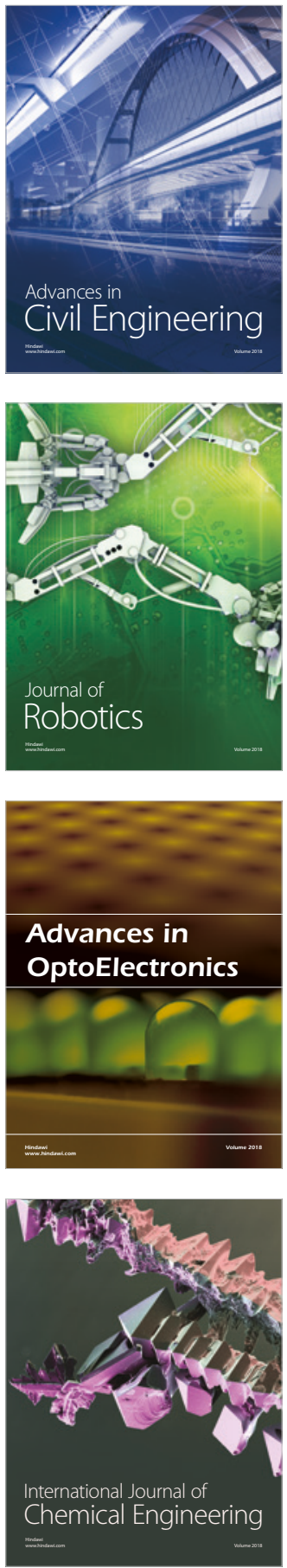

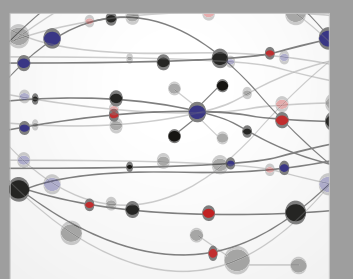

\section{Rotating \\ Machinery}

The Scientific World Journal

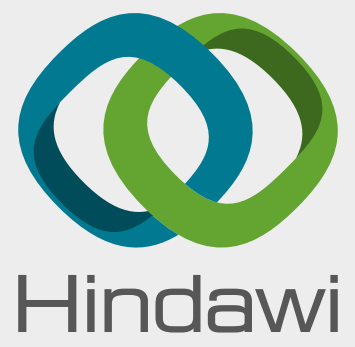

Submit your manuscripts at

www.hindawi.com
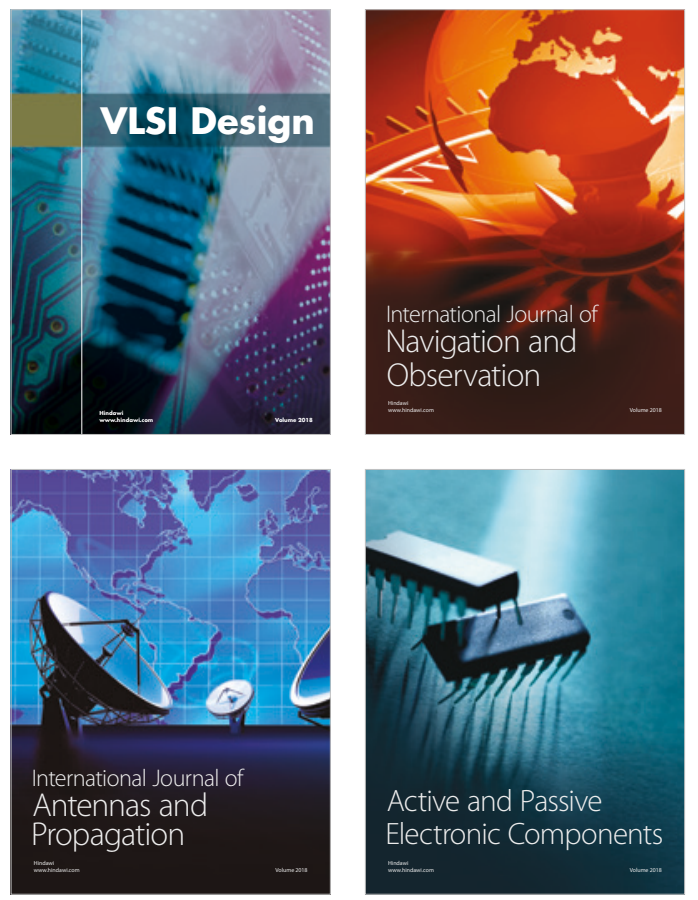
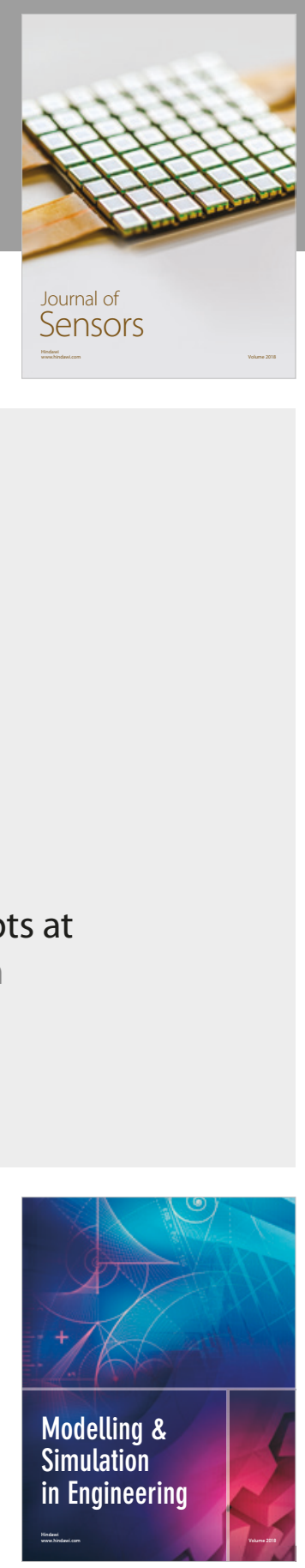

\section{Advances \\ Multimedia}
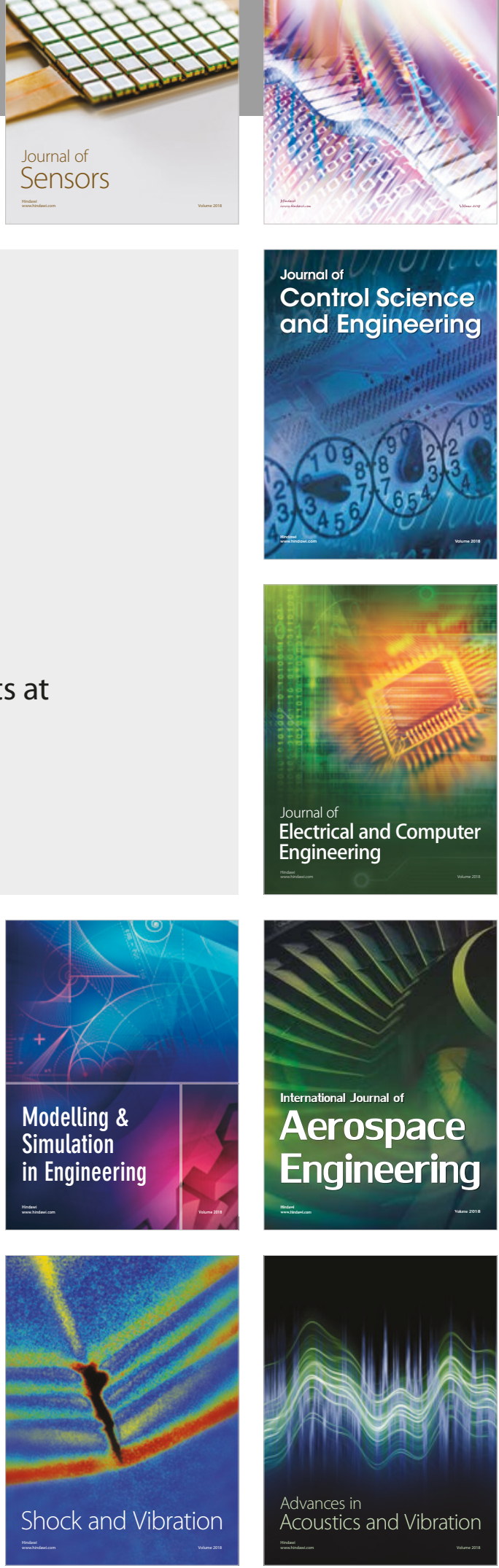